\title{
Acoustic eigenvalue analysis using a fast boundary element approach and a contour integral method
}

\author{
C.-J. Zheng ${ }^{1}$, H.-F. Gao ${ }^{2}$, T. Matsumoto ${ }^{2} \&$ H.-B. Chen ${ }^{1}$ \\ ${ }^{1}$ University of Science and Technology of China, China \\ ${ }^{2}$ Nagoya University, Japan
}

\begin{abstract}
In this paper, a contour integral method called the block Sakurai-Sugiura (SS) method is implemented to solve eigenvalue problems governed by the Helmholtz equation and formulated through the boundary element method. In the SS method, the nonlinear eigenvalue problem is converted into a standard linear eigenvalue problem, and eigenvalues whose multiplicity is larger than one can also be extracted by using the block version of the SS method. In order to solve the boundary element linear equations with multiple right-hand sides efficiently, a block IDR $(s)$ solver and an adaptive cross approximation are employed to improve the overall computational efficiency. Numerical examples are given to demonstrate the accuracy and effectiveness of the proposed method.

Keywords: eigenvalue analysis, contour integral, boundary element method, adaptive cross approximation, block $\operatorname{IDR}(s)$.
\end{abstract}

\section{Introduction}

The analysis of acoustic behavior of structures is usually very important. Besides acoustic radiation and scattering analyses, the eigenvalue analysis is also a very significant issue in engineering applications, for instance, in the vibration analysis of mechanical structures, such as automobiles and aircraft. As it is difficult to obtain analytical solutions for many engineering problems, numerical methods including the finite element method (FEM) and boundary element method (BEM) have become very powerful tools. 
Because of its high accuracy, reduction of dimensionality and superiority in solving infinite or semi-infinite wave propagation problems, the BEM has been widely applied for solving acoustic problems. However, the boundary element discretization leads to an asymmetric and dense coefficient matrix. As for the conventional BEM (CBEM), both the setting up and storage requirements of the matrix are of $O\left(n^{2}\right)$, where $n$ denotes the number of degrees of freedom (DOF). Also, direct solvers require $O\left(n^{3}\right)$ operations while iterative solvers $O\left(n^{2}\right)$. The high solution cost had constrained the extensive use of the BEM in largescale engineering applications for several decades. Moreover, as the coefficient matrix involves the wave number implicitly, the original eigenvalue problem for the Helmholtz equation becomes a nonlinear eigenvalue problem (NEP) when formulated by the BEM.

To tackle the difficulty of high solution cost, a number of techniques have been proposed. The fast multipole method (FMM) [1] is a very effective technique which improves the BEM efficiency [2-5]. However, the main disadvantage of the FMM is that the knowledge of the kernel expansion is required and the computational procedure is modified quite considerably in comparison with a CBEM code. A powerful alternative to the FMM is the adaptive cross approximation (ACA) [6]. The basic idea of the ACA is to divide the whole matrix into two rank (low- and full-rank) blocks, and the solution of linear system of equations is accelerated by calculating only a few entries of the matrix. In order to solve the NEP formulated by the BEM, contour integral methods have emerged. In this paper, a contour integral method called the block Sakurai-Sugiura (SS) method [7] is adopted, which can extract eigenvalues inside a given contour while preserving their multiplicity. In order to solve the boundary element linear equations with multiple right-hand sides efficiently, a block $\operatorname{IDR}(s)$ solver [8] and an ACA approach are employed to improve the overall computational efficiency.

\section{Boundary element formulations}

The Helmholtz equation which is the governing equation in steady-state linear acoustics can be reformulated into a boundary integral equation (BIE) defined on the boundary $\Gamma$ as follows:

$$
c(x) p(x)+\int_{\Gamma} q^{*}(x, y) p(y) \mathrm{d} \Gamma(y)=\int_{\Gamma} p^{*}(x, y) q(y) \mathrm{d} \Gamma(y)+p_{i}(x),
$$

where $c(x)$ is $1 / 2$ if $\Gamma$ is smooth around the source point $x, p(x)$ is the sound pressure, $p_{i}(x)$ is the incident wave, $q(y)$ and $q^{*}(x, y)$ are the normal derivatives of $p(y)$ and $p^{*}(x, y)$, and $y$ is the field point. The fundamental solution $p^{*}(x, y)$ is given as

$$
p^{*}(x, y)= \begin{cases}\frac{\mathrm{i}}{4} H_{0}^{(1)}(k r), & \text { for 2D problems } \\ \frac{\mathrm{e}^{\mathrm{i} k r}}{4 \pi r}, & \text { for 3D problems }\end{cases}
$$


where $\mathrm{i}$ is the imaginary unit, $k$ the wave number, $r=|y-x|$, and $H_{0}^{(1)}$ the Hankel function of the first kind. The boundary conditions are given as

$$
\begin{gathered}
p(x)=\bar{p}(x), \quad \text { on } \Gamma_{p}, \\
q(x)=\mathrm{i} \rho \omega \bar{v}(x), \quad \text { on } \Gamma_{q}, \\
p(x)=z v(x), \quad \text { on } \Gamma_{z},
\end{gathered}
$$

where $\rho$ is the medium density, $\omega$ the angular frequency, $v(x)$ the normal velocity, $z$ the acoustic impedance. The quantities with overbars indicate given values on the boundary $\left(\Gamma=\Gamma_{p}+\Gamma_{q}+\Gamma_{z}\right)$.

Discretizing $\Gamma$ using $N$ (e.g., piecewise constant) surface elements leads to the following equation (e.g., for acoustic radiation problems):

$$
\frac{1}{2} p^{i}+\sum_{j=1}^{N} H^{i j} p^{j}=\sum_{j=1}^{N} G^{i j} q^{j},
$$

where

$$
\begin{aligned}
H^{i j} & =\int_{\Gamma_{j}} q^{*}(x, y) \mathrm{d} \Gamma(y), \\
G^{i j} & =\int_{\Gamma_{j}} p^{*}(x, y) \mathrm{d} \Gamma(y) .
\end{aligned}
$$

In Eqs. (7) and (8), $\Gamma_{j}$ represents the piecewise constant element $j$. Collecting Eq. (6) for all boundary source points and expressing them in matrix form yields the following linear algebraic equations:

$$
\mathbf{H p}=\mathbf{G q} .
$$

Equation (9) can be rearranged into the following form by applying the boundary conditions:

$$
\mathbf{A x}=\mathbf{y},
$$

where $\mathbf{A}$ is the coefficient matrix which is asymmetric and fully populated, $\mathbf{x}$ is the vector containing the unknown boundary pressures and fluxes, and $\mathbf{y}$ is obtained by multiplying the given boundary conditions by the corresponding columns of the $\mathbf{H}$ and $\mathbf{G}$ matrices. Eq. (10) is solved, all the unknown boundary values can then be obtained. Once this has been done, one can calculate the sound pressure at any internal point by using the discretized form of Eq. (1) with $c(x)=1$.

It is known that the BEM based on Eq. (1) fails to yield unique solutions for exterior acoustic problems at the eigenfrequencies of the associated interior problems [9]. The Burton-Miller method [10] can be applied to tackle this difficulty. However, strongly singular and hypersingular boundary integrals are found in the Burton-Miller formulation. Instead of using various singularity subtraction techniques, they can be evaluated directly and efficiently when the piecewise constant element discretization is employed [11]. 
The coefficient matrix $\mathbf{A}$ in Eq. (10) involves the wave number $k$ implicitly, therefore, we have the following NEP:

$$
\mathbf{A}(k) \mathbf{x}=\mathbf{0} .
$$

If there is nontrivial solution, we have

$$
\operatorname{det}[\mathbf{A}(k)]=0 .
$$

Equation (12) is a highly nonlinear transcendental equation in $k$, hence it is very difficult to solve analytically. In order to solve this nonlinear eigenvalue problem, a contour integral method called the block SS method [7] is introduced in the next section.

\section{Eigenvalue analysis using block SS method}

The block SS method proposed by Asakura et al. [7] is a projection method which can extract eigenvalues inside a given contour $C$ while preserving their multiplicity. In this method, the moment matrices are defined as

$$
\mathbf{M}_{m}=\frac{1}{2 \pi \mathrm{i}} \int_{C} z^{m} \mathbf{U}^{H} \mathbf{A}(z)^{-1} \mathbf{V d} z
$$

where $\mathbf{U}$ and $\mathbf{V}$ can be taken as arbitrary nonzero $n \times L$ matrices, $n$ is the number of DOF of the problem, and $L$ should be superior to the maximum algebraic multiplicity of the eigenvalues lying inside $C$. The contour integral on $C$ can be approximated using the $N$-point trapezoidal rule. If the integral path is chosen as a circle with $C=\gamma+\rho \mathrm{e}^{\mathrm{i} \theta},(0 \leq \theta<2 \pi)$, we can obtain the shifted and scaled moment matrices as follows:

$$
\mathbf{M}_{m} \approx \hat{\mathbf{M}}_{m}=\frac{1}{N} \sum_{j=0}^{N-1}\left(\frac{\omega_{j}-\gamma}{\rho}\right)^{m+1} \mathbf{U}^{H} \mathbf{A}\left(\omega_{j}\right)^{-1} \mathbf{V}
$$

where $\omega_{j}=\gamma+\rho \mathrm{e}^{2 \pi \mathrm{i}(j+1 / 2) / N},(j=0,1, \cdots, N-1)$. Then, the Hankel matrices $\mathbf{H}_{1}$ and $\mathbf{H}_{2}$ can be formed as

$$
\begin{gathered}
\mathbf{H}_{1}=\left(\begin{array}{llll}
\mathbf{M}_{0} & \mathbf{M}_{1} & \ldots & \mathbf{M}_{K-1} \\
\mathbf{M}_{1} & \mathbf{M}_{2} & \ldots & \mathbf{M}_{K} \\
\vdots & \vdots & \ddots & \vdots \\
\mathbf{M}_{K-1} & \mathbf{M}_{K} & \ldots & \mathbf{M}_{2 K-2}
\end{array}\right), \\
\mathbf{H}_{2}=\left(\begin{array}{llll}
\mathbf{M}_{1} & \mathbf{M}_{2} & \ldots & \mathbf{M}_{K} \\
\mathbf{M}_{2} & \mathbf{M}_{3} & \ldots & \mathbf{M}_{K+1} \\
\vdots & \vdots & \ddots & \vdots \\
\mathbf{M}_{K} & \mathbf{M}_{K+1} & \ldots & \mathbf{M}_{2 K-1}
\end{array}\right) .
\end{gathered}
$$


where $K$ is a positive integer. It is proved that the eigenvalues of the matrix pencil $\mathbf{H}_{2}-k \mathbf{H}_{1}$ are identical to those of the original eigenvalue problem [7]. After obtaining $\mathbf{H}_{1}$ and $\mathbf{H}_{2}$, we perform singular value decomposition (SVD) on $\mathbf{H}_{1}$ as follows:

$$
\mathbf{H}_{1}=\mathbf{C} \boldsymbol{\Sigma} \mathbf{E}^{H}
$$

where $\boldsymbol{\Sigma}=\operatorname{diag}\left(\sigma_{1}, \sigma_{2}, \cdots, \sigma_{K L}\right)$. Hence, the original nonlinear eigenvalue problem can be converted into a standard eigenvalue problem of finding the eigenvalues $\lambda_{j}$ of the following matrix:

$$
\mathbf{B}=\mathbf{C}^{H} \mathbf{H}_{2} \mathbf{E} \boldsymbol{\Sigma}^{-1}
$$

After obtaining the $\lambda_{j}$, the eigenvalues $k_{j}$ can be recovered by

$$
k_{j}=\gamma+\rho \lambda_{j}
$$

Moreover, to ensure appropriate numerical accuracy, a suitable threshold can be found according to Ref. [12] to truncate the SVD in Eq. (17) in order to omit the small and unexpected eigenvalues.

It should be noted that we have to calculate $\mathbf{A}^{-1} \mathbf{V}$ for $N$ wave numbers along the integral path $C$ in Eq. (14). Instead of evaluating $\mathbf{A}^{-1}$, we can solve the following equations:

$$
\mathbf{A X}=\mathbf{V}
$$

where $\mathbf{X}$ and $\mathbf{V}$ are matrices of $n \times L$. It is known that Eq. (20) is very timeconsuming to obtain and solve in a conventional way even with appropriate iterative solvers. In order to accelerate the solution process and reduce the storage requirements, the ACA algorithm is introduced in the next section.

\section{ACA-BEM}

It is well known that the coefficient matrix of the BEM is neither singular nor rankdeficient except at the resonances. Because of the nature of the Green's function, however, the coefficient matrix consists of many numerically rank-deficient subblocks [6]. Namely, a boundary element matrix can be split into a collection of blocks, some of which, called low-rank blocks, allow a special compressed representation, while others, called full-rank blocks are computed directly. In the ACA-BEM, the low-rank blocks describe remote interactions of groups of boundary elements, and the ACA algorithm [6] allows to approximate the lowrank blocks using only few rows and columns of the blocks. For instance, let a rectangular matrix $\mathbf{Z}^{s \times t}$ represent a low-rank block in a boundary element coefficient matrix. The ACA algorithm aims to approximate $\mathbf{Z}^{s \times t}$ by $\tilde{\mathbf{Z}}^{s \times t}$, which 
can be written in the following product form:

$$
\mathbf{Z}^{s \times t} \approx \tilde{\mathbf{Z}}^{s \times t}=\mathbf{U}^{s \times r} \mathbf{V}^{r \times t}=\sum_{i=1}^{r} \mathbf{u}_{i}^{s \times 1} \mathbf{v}_{i}^{1 \times t}
$$

where $r$ is the number of ACA iterations and also the rank of the matrix $\tilde{\mathbf{Z}}^{s \times t}$, $\mathbf{U}^{s \times r}$ and $\mathbf{V}^{r \times t}$ are two dense rectangular matrices. The goal of the ACA algorithm is to achieve

$$
\left\|\mathbf{Z}^{s \times t}-\tilde{\mathbf{Z}}^{s \times t}\right\| \leq \epsilon\left\|\mathbf{Z}^{s \times t}\right\|
$$

for a given tolerance $\epsilon$, where $\|\cdot\|$ denotes the matrix Frobenius norm. In numerical applications, the rank $r \leq \min (s, t)$, thus, instead of storing and calculating all $s \times t$ entries, the algorithm only requires to calculate and store $r \times(s+t)$ entries. Then, the matrix-vector product can be evaluated in two steps as follows:

$$
\mathbf{Z}^{s \times t} \mathbf{X}^{t \times 1} \approx \tilde{\mathbf{Z}}^{s \times t} \mathbf{X}^{t \times 1}=\mathbf{U}^{s \times r}\left(\mathbf{V}^{r \times t} \mathbf{X}^{t \times 1}\right)
$$

Therefore, the number of essential operations for matrix-vector product is also $O(r(s+t))$. In the ACA-BEM, we first calculate the matrix and known vector product on the right-hand side of Eq. (10), then calculate the matrix and unknown vector product on the left-hand side and update the unknown vector in iterative solvers, such as the $\operatorname{IDR}(s)$ solver [13] adopted in this study. Since the boundary element linear equations (Eq. (20)) required in the block SS method are with multiple right-hand sides, a block version of $\operatorname{IDR}(s)$ solver [8] is employed. In order to further improve the computational efficiency, a block diagonal preconditioner [14] which is very simple and effective is also adopted.

\section{Numerical examples}

The rectangular box, as depicted in Fig. 1, is used as a numerical example to demonstrate the accuracy and efficiency of the proposed method. The sound pressures on the left and right side surfaces are given as $p_{L}$ and $p_{R}$, respectively. The other four surfaces are assumed to be rigid walls, i.e., normal velocities are specified as zero on them. The media of acoustic fields are assumed to be air with the density of $\rho=1.2 \mathrm{~kg} / \mathrm{m}^{3}$ and the sound speed of $C_{s}=340.0 \mathrm{~m} / \mathrm{s}$.

Piecewise constant triangular elements are applied to discretize the boundary surfaces, and all boundary integrals are evaluated numerically by using the Gaussian quadrature formula with 10 integration points. The ACA tolerance $\epsilon$ is set to $10^{-7}$. The iterative solver IDR(4) terminates iterations when the residue is below a tolerance of $10^{-7}$. All computations were carried out in double precision arithmetic on a desktop PC with an Intel $3.50 \mathrm{GHz}$ Core processor and $31.1 \mathrm{~GB}$ memory. 


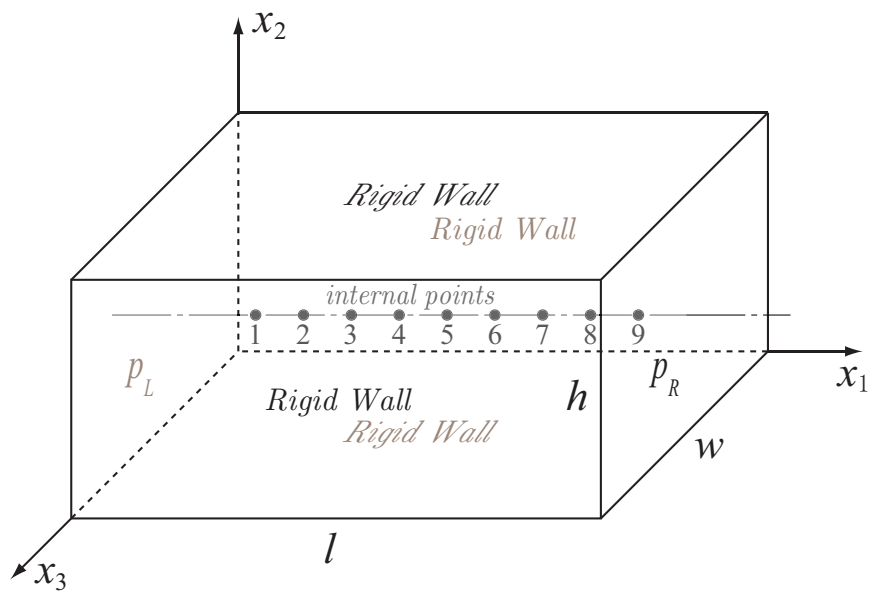

Figure 1: The rectangular box model.

\subsection{Convergence and efficiency studies}

In the numerical experiments, $l=w=h=1.0 \mathrm{~m}, p_{L}=0$ and $p_{R}=100.0 \mathrm{~Pa}$. Thus, the analytical sound pressure at an internal point $x\left(x_{1}, x_{2}, x_{3}\right)$ is given as

$$
p(x)=\frac{100 \sin \left(k x_{1}\right)}{\sin (k l)}, \quad 0 \leq x_{1} \leq l .
$$

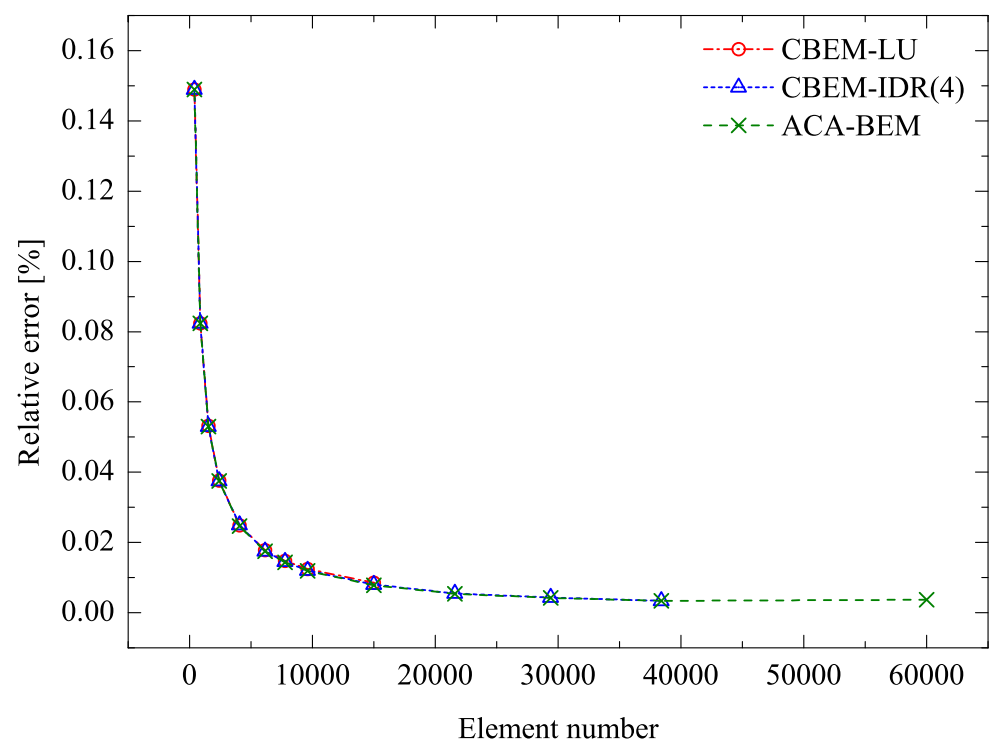

Figure 2: Relative errors of the numerical results. 


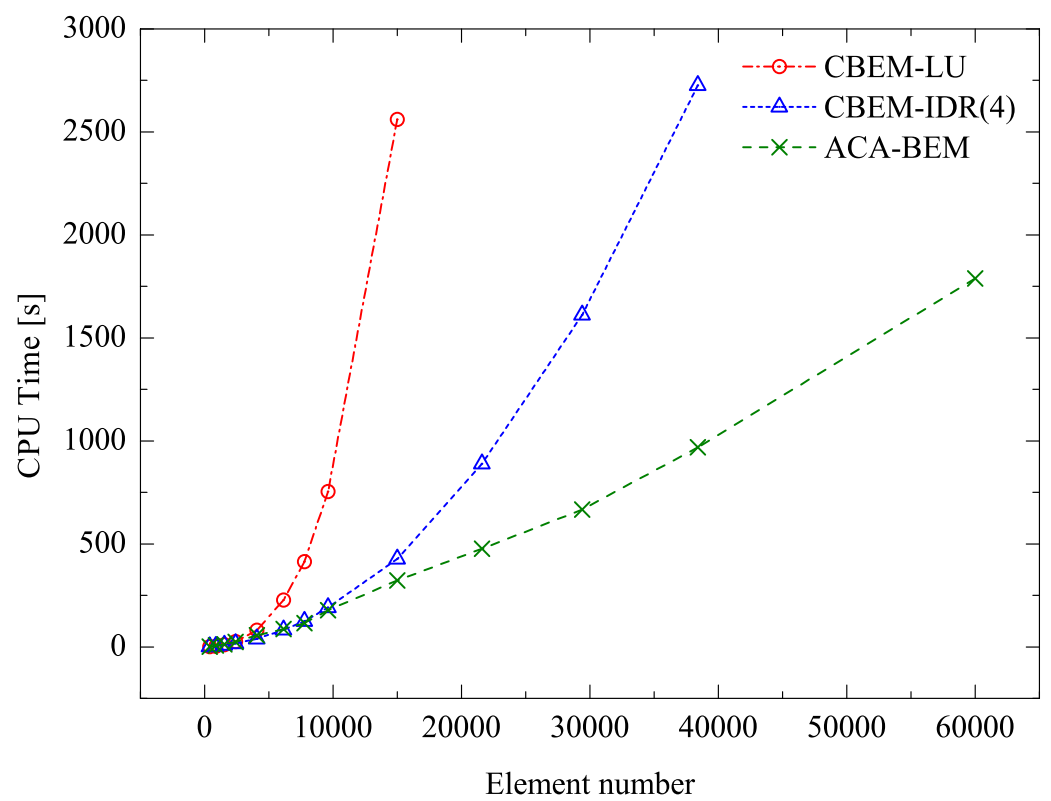

Figure 3: CPU times used to solve the box model.

The sound pressures at nine internal points on the central line of the box are calculated and compared with the analytical solution for a wave number $k=1.0 \mathrm{~m}^{-1}$. The relative errors of the results of different BEM approaches are compared in Fig. 2, where the relative errors are the mean percentage relative errors at the nine internal points. We refer to CBEM-LU and CBEM-IDR(4) as the results obtained by the CBEM with the LU-decomposition and IDR(4) solvers, respectively. It is shown that the ACA-BEM solutions are very close to those of the CBEM-LU and CBEM-IDR(4), and converge very rapidly. It is also known from Fig. 3 that the ACA-BEM approach is faster than the CBEM-LU and CBEMIDR(4) for models with more than 2400 and 7776 elements.

\subsection{Eigenvalue analysis}

In the eigenvalue analysis, by taking $C=\gamma+\rho \mathrm{e}^{\mathrm{i} \theta}, \gamma=(5.5,0), \rho=2.4$ to define the integral path, we obtain six eigenvalues located in the domain as shown in Table 1. The parameters in the block SS method are set as $N=256, K=$ $4, L=15$. The numerical results are given in Table 2, and the Error is defined as

$$
\text { Error }=\left|\frac{k_{\text {anal }}^{i}-\operatorname{Re}\left(k_{\text {num }}^{i}\right)}{k_{\text {anal }}^{i}}\right| \times 100(\%),
$$

where $k_{\text {anal }}^{i}$ and $k_{\text {num }}^{i}$ denote the analytical and numerical results, respectively. $\operatorname{Re}\left(k_{\text {num }}^{i}\right)$ indicates the real part of $k_{\text {num }}^{i}$.

It is found that the eigenvalues whose multiplicity is one or larger than one can both be extracted by using the proposed approach for acoustic wave problems. 
Table 1: Analytical eigenvalues from 3.1 to $7.9\left(\left[\mathrm{~m}^{-1}\right]\right)$.

\begin{tabular}{ccc}
\hline$\sqrt{n_{1}^{2}+n_{2}^{2}+n_{3}^{2}}$ & $k_{\text {anal }}^{i}$ & Multiplicity \\
\hline 1 & 3.14159 & 1 \\
$\sqrt{2}$ & 4.44288 & 2 \\
$\sqrt{3}$ & 5.44140 & 1 \\
2 & 6.28319 & 1 \\
$\sqrt{5}$ & 7.02481 & 4 \\
$\sqrt{6}$ & 7.69530 & 3 \\
\hline
\end{tabular}

Table 2: Numerical eigenvalues from 3.1 to $7.9\left(\left[\mathrm{~m}^{-1}\right]\right)$.

\begin{tabular}{cccccc}
\hline & \multicolumn{2}{c}{ CBEM } & & & \multicolumn{2}{c}{ ACA-BEM } \\
\cline { 2 - 3 } \cline { 5 - 6 }$i$ & $k_{i}$ & Error & & $k_{i}$ & Error \\
\hline 1 & $3.14628+1.38 \times 10^{-3}$ & 0.1493 & & $3.14628+1.38 \times 10^{-3}$ & 0.1493 \\
\hline 2 & $4.44730+9.00 \times 10^{-4}$ & 0.0995 & & $4.44730+9.01 \times 10^{-4}$ & 0.0995 \\
\hline 3 & $4.44730+9.00 \times 10^{-4}$ & 0.0995 & & $4.44732+8.93 \times 10^{-4}$ & 0.0999 \\
4 & $5.44652-6.50 \times 10^{-4}$ & 0.0941 & & $5.44654-6.27 \times 10^{-4}$ & 0.0945 \\
\hline 5 & $6.29129+1.90 \times 10^{-3}$ & 0.1289 & & $6.29128+1.92 \times 10^{-3}$ & 0.1288 \\
\hline 6 & $7.02711-1.16 \times 10^{-3}$ & 0.0327 & & $7.02711-1.16 \times 10^{-3}$ & 0.0327 \\
\hline 7 & $7.02849-1.36 \times 10^{-3}$ & 0.0524 & & $7.02849-1.36 \times 10^{-3}$ & 0.0524 \\
\hline 8 & $7.03485+1.75 \times 10^{-3}$ & 0.1429 & & $7.03484+1.75 \times 10^{-3}$ & 0.1428 \\
\hline 9 & $7.03485+1.75 \times 10^{-3}$ & 0.1429 & & $7.03485+1.75 \times 10^{-3}$ & 0.1429 \\
\hline 10 & $7.69976-3.89 \times 10^{-3}$ & 0.0580 & & $7.69976-3.89 \times 10^{-3}$ & 0.0580 \\
\hline 11 & $7.69976-3.89 \times 10^{-3}$ & 0.0580 & & $7.69976-3.89 \times 10^{-3}$ & 0.0580 \\
12 & $7.70763+6.65 \times 10^{-4}$ & 0.1602 & & $7.70762+6.66 \times 10^{-4}$ & 0.1601 \\
\hline
\end{tabular}

Since the ACA algorithm is employed to accelerate the BEM, the proposed approach can be used to solve large-scale acoustic eigenvalue problems.

\section{Conclusions}

In this paper, the SS method has been employed to solve nonlinear eigenvalue problems governed by the Helmholtz equation and formulated through the BEM. 
As a block version of the SS method is adopted, eigenvalues can be extracted while preserving their multiplicity. In order to accelerate the solution process and reduce the storage requirements of the BEM, the ACA algorithm associated with a block IDR $(s)$ solver and a block-diagonal preconditioner is employed. Numerical examples have been presented to demonstrate the validity and efficiency of the proposed approach, and also its great potential in large-scale acoustic eigenvalue analysis.

\section{References}

[1] Greengard, L. and Rokhlin, V., A fast algorithm for particle simulations. J Comput Phys, 73, pp. 325-348, 1987.

[2] Nishimura, N., Fast multipole accelerated boundary integral equation methods. ASME Appl Mech Rev, 55, pp. 299-324, 2002.

[3] Liu, Y.J., Fast Multipole Boundary Element Method: Theory and Applications in Engineering, Cambridge University Press, New York, 2009.

[4] Zheng, C.J., Chen, H.B., Matsumoto, T. and Takahashi, T., Three dimensional acoustic shape sensitivity analysis by means of adjoint variable method and fast multipole boundary element approach. CMES, 79, pp. 1-30, 2011.

[5] Zheng, C., Matsumoto, T., Takahashi, T. and Chen, H., A wideband fast multipole boundary element method for three dimensional acoustic shape sensitivity analysis based on direct differentiation method. Eng Anal Bound Elem, 36, pp. 361-371, 2012.

[6] Bebendorf, M., and Rjasanow, S., Adaptive low-rank approximation of collocation matrices. Computing, 70, pp. 1-24, 2003.

[7] Asakura, J., Sakurai, T., Tadano, H., Ikegami, T. and Kimura, K., A numerical method for nonlinear eigenvalue problems using contour integrals. JSIAM Letters, 1, pp. 52-55, 2009.

[8] Du, L., Sogabe, T., Yu, B., Yamamoto, Y. and Zhang, S.-L., A block IDR(s) method for nonsymmetric linear systems with multiple right-hand sides. J Comput Appl Math, 235, pp. 4095-4106, 2011.

[9] Schenck, H.A., Improved integral formulation for acoustic radiation problems. J Acoust Soc Am, 44, pp. 41-58, 1968.

[10] Burton, A.J. and Miller, G.F., The application of integral equation methods to the numerical solution of some exterior boundary-value problems. Proc Roy Soc Lond A, 323, pp. 201-210, 1971.

[11] Zheng, C., Matsumoto, T., Takahashi, T. and Chen, H., Explicit evaluation of hypersingular boundary integral equations for acoustic sensitivity analysis based on direct differentiation method. Eng Anal Bound Elem, 35, pp. 12251235, 2011.

[12] Gao, H.F., Matsumoto, T., Takahashi, T. and Isakari, H., Eigenvalue analysis for acoustic problem in 3D by boundary element method with the block Sakurai-Sugiura method. Eng Anal Bound Elem, 37, pp. 914-923, 2013. 
[13] Sonneveld, P. and Gijzen, M.B., IDR $(s)$ : a family of simple and fast algorithms for solving large nonsymmetric systems of linear equations. SIAM J Sci Comput, 31, pp. 1035-1062, 2008.

[14] Chen, K., and Harris, P.J., Efficient preconditioners for iterative solution of the boundary element equations for the three-dimensional Helmholtz equation. Appl Numer Math, 36, pp. 475-489, 2001. 